\title{
Effect of numerical diffusion on the water mass transformation in eddy-resolving models
}

\author{
L. Shogo Urakawa*, Hiroyasu Hasumi \\ Atmosphere and Ocean Research Institute, University of Tokyo, 5-1-5 Kashiwanoha, \\ Kashiwa, Chiba 277-8568, Japan
}

\begin{abstract}
This study investigates the effect of numerical diffusion associated with advection schemes on water mass transformation in an eddy-resolving model. The effect of numerical diffusion is evaluated as a residual between the total water mass transformation and the explicit water mass transformation: the former is calculated as the sum of meridional streamfunction and the temporal change rate of an isopycnal surface depth, and the latter is directly calculated with the use of the tendency equation of density. This method is used for investigating a dependency of numerical diffusion on explicit diffusivity. It is found that idealized channel experiments are categorized into three regimes according to a magnitude of explicit diffusivity: numerical diffusion, transitional, and explicit diffusion regimes. The numerical diffusion regime is defined as the regime where explicit diffusion changes do not significantly impact the solution. The magnitude of numerical diffusion is independent of the explicit diffusivity there. In the transitional regime, explicit (numerical) diffusion works more (less) with higher explicit diffusivity. Explicit and numerical diffusions are comparably important there. The explicit diffusion becomes significantly large and the numerical diffusion is almost negligible in
\end{abstract}

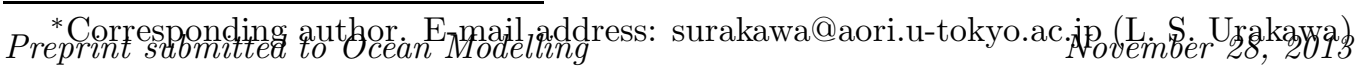


the explicit diffusion regime. The total diffusion effect on water mass transformation there is considerably larger than those in the two other regimes.

Two experiments are conducted with a Southern Ocean model under a realistic configuration. These belong to the numerical diffusion and transitional regimes. The model becomes a little too diffusive in the latter experiment. This result and results of channel experiments indicate that it is not an adequate option for a realistic Southern Ocean simulation that we adopt a diffusion coefficient in the explicit diffusion regime in order to reduce levels of numerical diffusion. It indicates that numerical diffusion is inevitable for eddy-resolving models with horizontal resolution around 0.1 degree and we must adequately evaluate its effect on model results when analyzing outputs of such high resolution models. The method proposed in the current study for assessing numerical diffusion will be useful for investigating an eddying ocean with numerical models.

Keywords: Eddy-resolving model, The Southern Ocean, Water mass transformation, Numerical diffusion

\section{Introduction}

High resolution modeling has become a widely available method to investigate oceanic phenomena. Numerical errors generally depend on grid spacing, so they are expected to be reduced by employing higher resolution. However, numerical errors depend also on other factors, and high resolution does not automatically guarantee reduction of errors. Using idealized simulations, Griffies et al. (2000) make a caveat about the influence of spurious mixing in high resolution modeling: large velocities resolved by models with high 
resolution induce large numerical diffusion. Lee et al. (2002) show their eddypermitting model actually suffers from that problem although they employ a relatively high-accuracy third-order advection algorithm. They suggest that high-resolution ocean models require less diffusive advection schemes. Hill et al. (2012) indicate that a very high-accuracy advection scheme developed by Prather (1986) involves little spurious diapycnal mixing in an interior region away from model boundaries with the use of an idealized channel model. However, it is still an open question whether such a state-of-the-art scheme reduces levels of numerical errors enough for a realistic ocean modeling. Urakawa \& Hasumi (2012) (UH hereafter) indicate that their eddyresolving Southern Ocean modeling is still not free from these numerical errors although their model adopts Prather's (1986) scheme. This problem should be further investigated with the use of high-resolution models.

Eddy resolving models usually adopt a horizontal biharmonic diffusion scheme as a way to parameterize mixing by sub-grid scale motions. Its diffusivity coefficient is, however, physically ${ }^{1}$ unknown and ranges widely from zero to $10^{10} \mathrm{~m}^{4} \mathrm{~s}^{-1}$ among eddy-resolving models of preceding studies which have horizontal resolution similar to that of the model in UH (Masumoto et al., 2004; Lee et al., 2007; Tanaka \& Hasumi, 2008a,b). It is anticipated that the stronger sub-grid scale eddy diffusivity results in the smoother distributions of tracers. An advection scheme tends to cause less numerical

\footnotetext{
${ }^{1}$ Strictly speaking, the use of biharmonic diffusion is not physically justified. It is commonly used in high-resolution models in order to dissipate tracer variances in the small scale and prevent models to blow up. However, there must be a "physically" adequate value for its coefficient as long as it tries to represent mixing by sub-grid scale processes.
} 
errors in that case. Reproducibility of models is also expected to depend on the explicit diffusivity ${ }^{2}$. Therefore, we need to know an adequate choice for the explicit diffusivity and the effect of numerical errors on a model's solution in eddy resolving modeling. We try to address this problem by conducting eddy resolving simulations of the Southern Ocean.

The current study shows that mixing induced by numerical errors associated with an advection scheme works in the same way as an explicit horizontal biharmonic diffusion. So, it is called "numerical diffusion" hereafter although numerical diffusion is not the sole source of numerical errors to be exact. This study also reveals that numerical diffusion inevitably becomes comparable to or larger than explicit diffusion even with a very high-accuracy advection scheme when the explicit diffusivity is adjusted to reproduce realistic stratification of the Southern Ocean, especially near the abyss, as long as we use a eddy resolving model with horizontal resolution around 0.1 degree. So, we must adequately evaluate it when analyzing outputs of such a highresolution model. Hill et al. (2012) and Ilıcak et al. (2012) utilize a temporal change of minimum potential energy to quantify the magnitude of numerical diffusion. This method gives us effective diffusivity (diffusivity induced by both explicit and numerical diffusions) but cannot isolate numerical diffusion in experiments with external forcings and/or explicit diffusions. Burchard \& Rennau (2008) propose another method in which the magnitude of numerical diffusion is evaluated as the difference of decay rate between the advected

\footnotetext{
${ }^{2}$ Explicit diffusivity denotes diffusion coefficients for explicit diffusion schemes. Explicit diffusion means diffusions explicitly implemented in a model. In other words, they are diffusions which are not numerical diffusion associated with an advection scheme.
} 
square of a tracer and the square of an advected tracer. It is compared to the turbulent mean tracer variance decay rate calculated with explicit diffusivity and spatial gradients of the tracer. This decay rate defines the magnitude of explicit diffusion. We can compare these two decay rates with each other and tell which of explicit and numerical diffusions is the larger. However, the tracer variance decay does not clearly show how diffusions quantitatively affect a model's solution such as a density distribution and a circulation. The main subject of the current study is to develop a way to quantitatively investigate the effect of numerical diffusion associated with an advection scheme on a tracer field.

We utilize the idea of water mass transformation, which has been developed by many studies (e.g., Walin, 1982). The water mass is one of the basic concepts in physical oceanography. It is important to know how water masses change their property when studying diabatic circulation. Moreover, this approach enables us to investigate the way how numerical diffusion quantitatively affects the density field in the ocean. We discuss a relationship between water mass transformation and meridional overturning circulation as is done by Lee et al. (2002) and propose a way to diagnose the effect of numerical diffusion on water mass transformation rate there.

The remainder of this paper is composed of the following sections. Section 2 shows an experimental design of this study. Because an eddy-resolving model of the Southern Ocean requires a lot of computational resource, it is difficult to carry out a sensitivity study on explicit horizontal diffusivity with it. We conduct only two experiments with different explicit horizontal diffusion coefficients in the current study. Results of these experiments 
are complemented by idealized channel experiments. Section 3 introduces methodology for analysis in the current study. Sections 4 and 5 show results of numerical experiments with the Southern Ocean model and the idealized channel model in the current study, respectively. Section 6 gives conclusions and discussions.

\section{Experimental design}

The model employed for this study is COCO, version 4 (Hasumi, 2006). This model is based on the primitive equations under the hydrostatic and Boussinesq approximations with explicit free surface. It is formulated on a general orthogonal curvilinear horizontal coordinate and the geopotential height vertical coordinate. This model is run under two different configurations, which is briefly summarized in Table 1. One is called the Southern Ocean model and the other is called the channel model, hereafter. Both models have horizontal resolution of $1 / 8^{\circ}$ in longitude and $1 / 12^{\circ}$ in latitude. An enstrophy-conserving scheme (Ishizaki \& Motoi, 1999) and a biharmonic friction with Smagorinsky-like viscosity $(\mathrm{C}=2.5$; Griffies \& Hallberg, 2000) are employed for momentum equations. The models adopt the second-order moment (SOM) scheme developed by Prather (1986) with the flux limiter of Morales Masqueda \& Holloway (2006), which is known to cause less numerical diffusion than conventionally used ones (Morales Masqueda \& Holloway, 2006). Vertical diffusion coefficient is $5 \times 10^{-5} \mathrm{~m}^{2} \mathrm{~s}^{-1}$. A horizontal biharmonic diffusion scheme is used there and its coefficient differs among experiments described below. Specific configurations for the two models are shown in following sections, respectively. 


\subsection{The Southern Ocean model}

The model domain is the Southern Ocean from $75^{\circ} \mathrm{S}$ to $20^{\circ} \mathrm{S}$. The northern and southern limits of the model domain are solid boundaries. A realistic bathymetry based on GEBCO (IOC, IHO and BODC, 2003) is used, and the coastline around Antarctica is modified with the use of MODIS Mosaic of Antarctica (Haran et al., 2005). Ice shelves and grounded icebergs in this model are treated as land. The model has 81 levels in the vertical direction, and the grid spacing is $50 \mathrm{~m}$ for the upper $2000 \mathrm{~m}$ and linearly increased to $100 \mathrm{~m}$ toward the bottom. Two different biharmonic horizontal diffusion coefficients are used in this study: $1 \times 10^{10} \mathrm{~m}^{4} \mathrm{~s}^{-1}$ and $5 \times 10^{7} \mathrm{~m}^{4} \mathrm{~s}^{-1}$. These experiments are hereafter called HIGH and LOW, respectively. The former experiment coincides with the experiment of UH.

The model is spun up for 1 year from a state of rest. During this spinup period, potential temperature and salinity are restored to WOCE global hydrographic climatology (WGHC; Gouretski \& Koltermann, 2004) from top to bottom with a restoring time of 30 days. Then, the model is forced for 10 years by monthly climatological average of Röske's (2001) wind forcing. Sea surface temperature and salinity are restored to the monthly climatological values of World Ocean Atlas 2001 (WOA'01; Stephens et al., 2002; Boyer et al., 2002) with a restoring time of 50 days. At the northern boundary, potential temperature and salinity from $23^{\circ} \mathrm{S}$ to $20^{\circ} \mathrm{S}$ are restored to WGHC from top to bottom with a restoring time of 30 days. Dense shelf water (DSW) on the Antarctic continental shelf is prescribed by restoring potential temperature and salinity in the area where the depth of the sea bottom is shallower than $1000 \mathrm{~m}$ depth to the monthly climatological average of a 
high-resolution East Antarctic (EAA) model (Kusahara et al., 2010, 2011). A restoring time is 30 days except for 5 days at the southern boundary of the model domain. A set of 3-day interval snapshots for the last 1 year is analyzed.

\subsection{Idealized channel model}

This model has a rectangular and flat bottom basin. The model size is $20^{\circ}$ in longitude, $20^{\circ}$ in latitude from $50^{\circ} \mathrm{S}$ to $30^{\circ} \mathrm{S}$ and $4000 \mathrm{~m}$ deep. Vertical resolution is $50 \mathrm{~m}$. Periodic boundary conditions are adopted in the zonal direction. The initial condition of potential temperature and salinity is zonally uniform. This initial state is taken from $\mathrm{WGHC}$ at $49^{\circ} \mathrm{E}$ in longitude and from $50^{\circ} \mathrm{S}$ to $30^{\circ} \mathrm{S}$ in latitude. Undefined values in WGHC are linearly interpolated with values of surrounding water. The initial meridional velocity is zero and the zonal velocity is calculated with the use of $u=-\frac{1}{f \rho_{0}} \frac{\partial p}{\partial y}$, where $\rho_{0}$ is a fixed reference density value $\left(10^{3} \mathrm{~kg} \mathrm{~m}^{-3}\right)$.

Two kinds of experiments are conducted with this model. One is a set of experiments where the model is integrated for 1 year without external forcings. These are called the short-run experiments hereafter. A linear equation of state (EOS) is also used in the former experiment as well as the nonlinear EOS. Horizontal biharmonic diffusion coefficients used there range from zero to $2 \times 10^{11} \mathrm{~m}^{4} \mathrm{~s}^{-1}$. The model is integrated for 40 years without explicit horizontal diffusion in the other kind of experiment and the last 1 year is analyzed. This experiment is called the long-run experiment. It is driven by a sinusoidal zonal wind, whose stress is 0 at the northern and southern boundaries and $0.1 \mathrm{~N} \mathrm{~m}^{-2}$ at $40^{\circ} \mathrm{S}$. Sea surface temperature and salinity are restored to those of initial values with a restoring time of 
Table 1: Configurations of experiments. NL and LI in the EOS column means nonlinear and linear, respectively.

\begin{tabular}{cccccc} 
Model & $\begin{array}{c}\text { Integration } \\
\text { period }\end{array}$ & $\begin{array}{c}\text { Horizontal } \\
\text { diffusivity } \\
{\left[\mathrm{m}^{4} \mathrm{~s}^{-1}\right]}\end{array}$ & $\begin{array}{c}\text { Vertical } \\
\text { diffusivity } \\
{\left[\mathrm{m}^{4} \mathrm{~s}^{-1}\right]}\end{array}$ & EOS & forcings \\
\hline The Southern Ocean & 11 years & $10^{10}$ and $5 \times 10^{7}$ & $5 \times 10^{-4}$ & NL & On \\
Channel (short-run) & 1 year & $0-2 \times 10^{11}$ & $5 \times 10^{-4}$ & NL and LI & Off \\
Channel (long-run) & 40 years & 0 & $5 \times 10^{-4}$ & NL & On
\end{tabular}

50 days. We analyze a set of 1-day interval snapshots for each experiment. Note that the results do not qualitatively and quantitatively change with the use of a set of 5-day interval snapshots.

\section{A method for diagnosing water mass transformations induced by numerical mixing}

We use the potential density referenced to 2000 dbar $\left(\sigma_{2}\right)$ in analysis of experiments with the nonlinear EOS. "Density" denotes the potential density hereafter. Let us consider an arbitrary isopycnal surface $S_{\sigma}$ whose density is equal to $\sigma$. The following relationship holds there:

$$
\frac{\partial}{\partial t} V(y, \sigma)=\Omega(y, \sigma)-\psi(y, \sigma)
$$

where $V(y, \sigma)$ describes volume contained in the layer below $S_{\sigma}$ and to the south of $y$. The left hand side of Eq. 1 is calculated from the difference between the initial and final states of the analyzed 1 year period. $\Omega(y, \sigma)$ and $\psi(y, \sigma)$ describe a volume transport across $S_{\sigma}$ to the south of $y$ and a transport across $y$ of water below $S_{\sigma}$, respectively. Water masses are defined 
Table 2: Water mass definitions in this study. Density ranges are defined with the use of layer-average potential temperature and salinity at the hydrographics sections shown by Sloyan \& Rintoul (2001) (See their Table 2).

\begin{tabular}{rr}
\hline \hline Water masses & \multicolumn{1}{c}{$\sigma_{2}$} \\
\hline Surface/thermocline water (STW) & -34.70 \\
Subantarctic Mode Water (SAMW) & $34.70-35.75$ \\
Antarctic Intermediate Water (AAIW) & $35.75-36.30$ \\
Upper Circumpolar Deep Water (UCDW) & $36.30-36.86$ \\
Lower Circumpolar Deep Water (LCDW) & $36.86-36.98$ \\
Antarctic Bottom Water (AABW) & $36.98-$ \\
\hline
\end{tabular}

by density in this study (Table 2 ). Thus, $\Omega(y, \sigma)$ denotes a water mass transformation rate in the current study. A positive value denotes transformation toward a denser water mass. $\psi(y, \sigma)$ is called a meridional streamfunction here. It is an annual average of meridional streamfunctions at all snapshots which are calculated with the use of potential temperature, salinity and meridional velocity. $\Omega(y, \sigma)$ is also calculated as an annual average of water mass transformation rates at all snapshots which are calculated with the method described below. In a steady state, these water mass transformation rate and meridional streamfunction coincide with each other.

$\Omega(y, \sigma)$ can be calculated from the thermodynamic equations. The temporal and spatial derivatives of density, $\sigma$, are described as

$$
\begin{aligned}
& \frac{\partial \sigma}{\partial t}=\alpha^{*} \frac{\partial \theta}{\partial t}+\beta^{*} \frac{\partial s}{\partial t}, \\
& \nabla \sigma=\alpha^{*} \nabla \theta+\beta^{*} \nabla s,
\end{aligned}
$$

where $\theta$ and $s$ denote potential temperature and salinity, and $\alpha^{*}$ and $\beta^{*}$ are 
defined as: $\alpha^{*} \equiv \partial \sigma / \partial \theta$ and $\beta^{*} \equiv \partial \sigma / \partial s$. Note that $\nabla=(\partial / \partial x, \partial / \partial y, \partial / \partial z)$. Advection-diffusion equations for potential temperature and salinity in a model are described as:

$$
\begin{aligned}
& \frac{\mathrm{D} \theta}{\mathrm{D} t}=\mathcal{D}(\theta)+q_{\theta}, \\
& \frac{\mathrm{D} s}{\mathrm{D} t}=\mathcal{D}(s)+q_{s},
\end{aligned}
$$

where $\mathrm{D} / \mathrm{D} t$ denotes the material derivative and $\mathcal{D}(\phi)$ is the contribution of diffusion ${ }^{3}$ to the tendency of $\phi . q_{\theta}$ and $q_{s}$ are source/sink terms of potential temperature and salinity, respectively. They include effects of sea surface and lateral boundary conditions, and convective adjustment in the current study. Note that $\mathcal{D}(\phi)$ includes the effect of numerical diffusion associated with an advection scheme. The advection-diffusion equation for density can be derived as:

$$
\begin{aligned}
\frac{\mathrm{D} \sigma}{\mathrm{D} t} & =\alpha^{*} \mathcal{D}(\theta)+\beta^{*} \mathcal{D}(s)+\alpha^{*} q_{\theta}+\beta^{*} q_{s} \\
& =\mathcal{D}(\sigma)+\mathcal{C}+\alpha^{*} q_{\theta}+\beta^{*} q_{s}
\end{aligned}
$$

The contribution of diffusion terms in the first line is decomposed into the diffusion term of density $(\mathcal{D}(\sigma))$ and the cabbeling ${ }^{4}$ term $(\mathcal{C})$ in the second line (e.g., Urakawa \& Hasumi, 2009). The effects of diffusion and cabbeling

\footnotetext{
${ }^{3}$ It is known that the biharmonic diffusion could cause an anti-diffusive tracer flux (Delhez \& Deleersnijder, 2007). However, UH confirm that such an anti-diffusive process is scarcely found in our model. So, we use the term "diffusion" here and hereafter in the current study.

${ }^{4}$ Cabbeling is calculated as follows: $\mathcal{C}=-\nabla \alpha \cdot \mathcal{F}(\theta)-\nabla \beta \cdot \mathcal{F}(s)$, where $\mathcal{F}(\phi)$ denotes a diffusive flux of an arbitrary tracer $\phi$.
} 
can be decomposed into the explicit ones $\left(\mathcal{D}_{\text {exp }}, \mathcal{C}_{\text {exp }}\right)$ and numerical ones $\left(\mathcal{D}_{\text {num }}, \mathcal{C}_{\text {num }}\right)$. Then, the tendency equation for density can be rewritten as:

$$
\frac{\mathrm{D} \sigma}{\mathrm{D} t}=\mathcal{D}_{\text {exp }}(\sigma)+\mathcal{D}_{\text {num }}(\sigma)+\mathcal{C}_{\text {exp }}+\mathcal{C}_{\text {num }}+\alpha^{*} q_{\theta}+\beta^{*} q_{s} .
$$

The material derivative of density on the left hand side can be described as:

$$
\frac{\mathrm{D} \sigma}{\mathrm{D} t}=\omega_{\sigma} \nabla_{\perp} \sigma
$$

where $\omega_{\sigma}$ is the diapycnal velocity across $S_{\sigma}$ and $\nabla_{\perp} \sigma$ is the gradient of density in the direction normal to $S_{\sigma}$. The water mass transformation rate $\Omega$ can be calculated by integrating $\omega_{\sigma}$ over $S_{\sigma}$ :

$$
\Omega=\iint_{S_{\sigma}}\left(\mathcal{D}_{\text {exp }}(\sigma)+\mathcal{D}_{\text {num }}(\sigma)+\mathcal{C}_{\text {exp }}+\mathcal{C}_{\text {num }}+\alpha^{*} q_{\theta}+\beta^{*} q_{s}\right)\left(\nabla_{\perp} \sigma\right)^{-1} d S .
$$

$\Omega$ is a function of $y$ and $\sigma$ but its description is omitted hereafter in this section. The right hand side of this equation can be transformed with the use of a generalized form of Leibnitz theorem as (Iudicone et al., 2008):

$$
\begin{aligned}
\Omega & =\frac{\partial}{\partial \sigma} \iiint_{V}\left(\mathcal{D}_{\text {exp }}(\sigma)+\mathcal{D}_{\text {num }}(\sigma)+\mathcal{C}_{\text {exp }}+\mathcal{C}_{\text {num }}+\alpha^{*} q_{\theta}+\beta^{*} q_{s}\right) d V \\
& =\Omega_{D E}+\Omega_{D N}+\Omega_{C E}+\Omega_{C N}+\Omega_{Q}
\end{aligned}
$$

The first to fourth terms in the second line correspond to those in the parentheses of the first line in the same order and $\Omega_{Q}$ corresponds to the remaining two terms.

We can easily calculate every term in the second line of Eq. 10 except for water mass transformation rates due to numerical diffusion $\left(\Omega_{D N}\right.$ and $\left.\Omega_{C N}\right)$ with the use of representations in the model. Numerical cabbeling 
term $\Omega_{C N}$ can be directly estimated with the use of the method in Appendix A. Utilizing Eq. 1, we can indirectly diagnose $\Omega_{D N}$ by the following equation:

$$
\Omega_{D N}=\psi+\frac{\partial V}{\partial t}-\Omega_{D E}-\Omega_{C E}-\Omega_{C N}-\Omega_{Q} .
$$

Appendix B shows discrete representations of these terms.

Water masses tend to move not along an isopycnal surface but along a neutral surface. Water mass transformation rates diagnosed with the use of density does not perfectly represent what actually goes in a model and contains errors. This might affect conclusions of this study. We also conduct the idealized experiments with a linear EOS as already noted. Unique density surfaces can be defined there and do not suffer from the errors noted above. We confirm that our conclusions do not change there quantitatively and qualitatively.

\section{Results of the Southern Ocean model}

\subsection{Reproducibility of the experiments HIGH and LOW}

The meridional streamfunction $\psi$ shows three major cells in the Southern Ocean: a subtropical cell $\left(\sigma_{2}<35\right)$, a subpolar cell $\left(35<\sigma_{2}<36.7\right)$ and a deep cell $\left(36.7<\sigma_{2}\right)$ (Fig. 1). The subpolar cell is associated with the NADW formation and the deep cell is associated with the AABW formation. The spatial pattern and the magnitude of the subtropical cell differ little between the experiments HIGH and LOW. The magnitude of the subpolar cell is stronger in the experiment HIGH, whereas that of the deep cell is weaker therein.

This meridional streamfunction in each experiment largely differs from the net water mass transformation rate $\Omega$, which is calculated as a sum of $\psi$ 


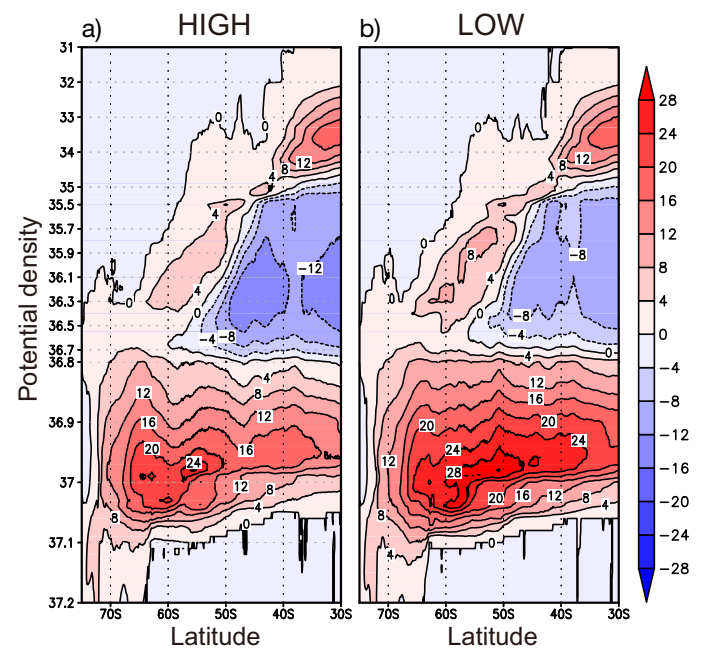

Figure 1: $\psi$ in (a) the experiment HIGH and (b) the experiment LOW. The contour interval is $4 \mathrm{~Sv}$. Positive for counter-clockwise.

and the volume inflation term $\partial V / \partial t$ (Fig. 2). The difference is especially large in the dense water mass classes $\left(\sigma_{2}>36.8\right)$. There is another clockwise cell between the deep cell and the ocean bottom. This cell means that the bottom water is modified into lighter water at the rate of about 40 and 25 $\mathrm{Sv}$ in the experiments HIGH and LOW, respectively.

$\Omega$ seemingly indicates a poleward transport of the densest water at the corresponding rate. This results from the fact that the meridional streamfunction can not strictly be defined in a non-steady state as already discussed by Marsh et al. (2000). $\psi$ shows the correct meridional volume transport because it is calculated from the meridional velocity, but inferred diapycnal volume transports are generally incorrect. Likewise, $\Omega$ offers the right information about the diapycnal volume transport, but inferred meridional transports are incorrect. For example, the net water mass transformation in the experiment HIGH (LOW) shows that about 30 (9) Sv of dense wa- 
ter is diapycnally transported toward the lighter water mass layer across the isopycnal surface of $37 \sigma_{2}$ to the south of $30^{\circ} \mathrm{S}$. It does not mean that the corresponding amount of the bottom water $(\sigma>37)$ is meridionally transported into the Southern Ocean at $30^{\circ} \mathrm{S}$ in our model. $\psi$ shows that over 4 (8) Sv of the bottom water is transported northward at $30^{\circ} \mathrm{S}$ in the experiment $\mathrm{HIGH}$ (LOW) (Fig. 1). There is a large difference between them which amounts to about 34 (17) Sv in the experiment HIGH (LOW). Such a difference between $\psi$ and $\Omega$ shows that the model is in a non-steady state. This is due to the short integration time. However, it is not an appropriate option to extend the model integration and achieve a steady state in order to fill this gap. As discussed in UH, this is not feasible from the standpoint of available numerical resources and leads to unrealistic distributions of potential temperature and salinity in this model. The experiments with the current model aim to quantitatively evaluate water mass transformation rates due to numerical diffusion in the realistic eddy-resolving simulations of the Southern Ocean. The realistic active tracer distributions and eddy activities are important for the current purpose. It is better to be based on such a non-steady but close-to-observed state in this study.

\subsection{Water mass transformation by explicit and numerical diffusions}

We separately calculate water mass transformation rates induced by explicit and numerical diffusions. UH show that explicit horizontal biharmonic diffusion in our model leads to strong diapycnal diffusion in the experiment HIGH (Fig. 3a). It modifies a significant amount of LCDW and AABW into lighter waters. On the other hand, there is hardly any explicit horizontal diffusion in experiment LOW (Fig. 3b). Note that the contour interval is 

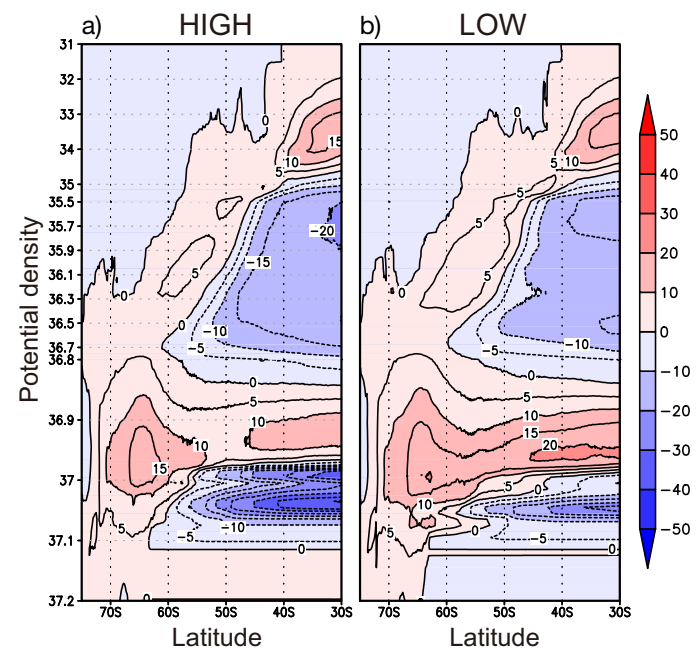

Figure 2: The same as Fig. 1 but $\Omega$. The contour interval is $5 \mathrm{~Sv}$.

different between Fig. 3a and b. Its magnitude is less than $1 \mathrm{~Sv}$ in most of the density range and the negative peak value is about $1.3 \mathrm{~Sv}$ found in the AABW class.

We diagnose the water mass transformation rate induced by numerical diffusion $\left(\Omega_{D N}\right)$ with the use of Eq. 11. Figure 4 shows water mass transformation induced by the total horizontal diffusion ${ }^{5}, \Omega_{D}^{H}$, which is the sum of $\Omega_{D N}$ and the horizontal part of $\Omega_{D E}\left(\Omega_{D E}^{H}\right)$. In the experiment HIGH, the pattern of this water mass transformation differs little from that of $\Omega_{D E}^{H}$ but its magnitude slightly increases in the dense LCDW and AABW classes. The negative peak value is about $-47 \mathrm{~Sv}$ for $\Omega_{D E}^{H}$ and about $-53 \mathrm{~Sv}$ for $\Omega_{D}^{H}$. Explicit

\footnotetext{
${ }^{5} \Omega_{D N}$ cannot be decomposed into its horizontal and vertical parts. As is discussed in $\mathrm{UH}$, however, the transformation rate due to numerical diffusion is quite similar to that due to horizontal diffusion. So, we believe that numerical diffusion occurs in the horizontal plane.
} 


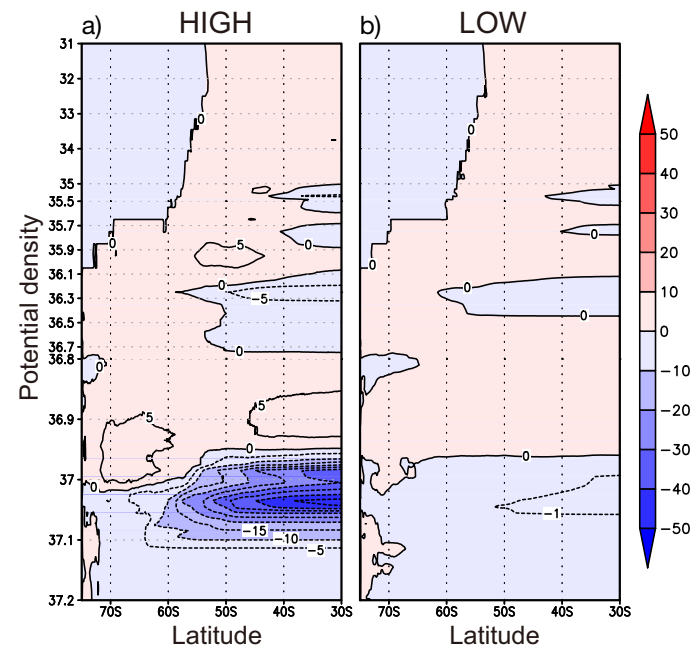

Figure 3: The same as Fig. 1 but $\Omega_{D E}^{H}$. The contour interval is $5 \mathrm{~Sv}$ for (a) and $1 \mathrm{~Sv}$ for (b).

horizontal diffusion dominates in water mass transformation but numerical diffusion is not negligible in the experiment HIGH. On the other hand, $\Omega_{D}^{H}$ is considerably dissimilar to $\Omega_{D E}^{H}$ in the experiment LOW. There appears strong modification to lighter waters in the dense LCDW and AABW classes as found in the experiment HIGH. It indicates that the numerical diffusion well imitates the explicit diffusion in the experiment LOW. Generally speaking, numerical diffusion could be anti-diffusive. Such up-gradient fluxes of active tracers lead to negative cabbeling effect on the water mass transformation, that is to say modification to lighter waters by cabbeling. However, the numerical cabbeling scarcely shows such a negative cabbeling effect in our model (not shown). The SOM scheme is known to hardly cause an anti-diffusive flux (Morales Masqueda \& Holloway, 2006).

The patterns of the water mass transformation due to the total diffusion 


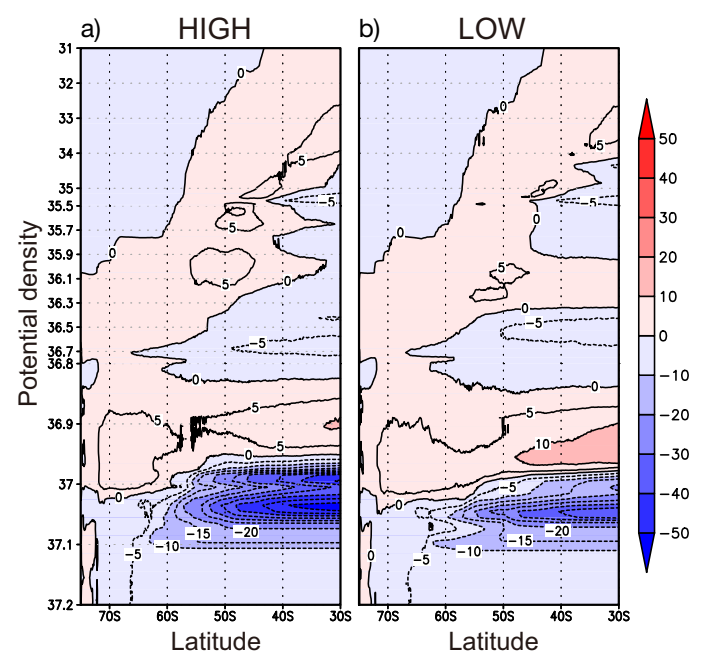

Figure 4: The same as Fig. 1 but the sum of $\Omega_{D N}$ and $\Omega_{D E}^{H}$. The contour interval is $5 \mathrm{~Sv}$.

in experiments HIGH and LOW are very similar. However, the magnitudes significantly differ from each other. UH discuss that strong modification of dense water in the experiment $\mathrm{HIGH}$, which amounts up to $53 \mathrm{~Sv}$, is too large for a realistic simulation of the Southern Ocean. The experiment LOW also shows the strong modification but it is quite moderate. UH show that this modification in the experiment LOW does not seem unrealistic when the insufficient dense water formation in the model is taken into account. The experiment LOW looks more realistic, whereas the experiment HIGH seems a little too diffusive.

Our two experiments HIGH and LOW seem to belong to two different regimes in terms of explicit horizontal diffusivity: one is a regime where explicit diffusion dominates but numerical diffusion is not negligible, and the other is a regime where numerical diffusion dominates and explicit diffusion is almost negligible. Water mass transformation induced by numerical diffu- 
sion as well as explicit diffusion seems to significantly depend on an explicit horizontal diffusion coefficient. Such a dependency has not well been investigated by preceding studies. It is difficult to carry out a sensitivity study on explicit horizontal diffusivity with the use of our eddy-resolving model of the Southern Ocean because of its high numerical cost. So, we utilize an idealized channel model and conduct numerical experiments with various explicit horizontal diffusion coefficients.

\section{Results of the channel model}

Figures $5 \mathrm{a}$ and $\mathrm{b}$ show the initial state of potential temperature and salinity for the channel model, respectively. A front exists around $40^{\circ} \mathrm{S}$ in both temperature and salinity profiles. Isothermal and isohaline surfaces are steeply inclined in the shallow region above $1000 \mathrm{~m}$ depth. Density also has a strong front around $42^{\circ} \mathrm{S}$ (Fig. 5c), although salinity inversely contributes to it. Strong baroclinicity of this initial condition leads to instability which generates mesoscale eddies in our channel model (Fig. 5d). These eddies meridionally transport heat and salinity and restratify the steep isopycnal front found in the initial state (Fig. 5e). The meridional streamfunction shows an anticlockwise circulation which pushes the isopycnal surface down in the south and pulls them up in the north (Fig. 5e). This streamfunction is not along the density surfaces because our short-run experiments are not in a steady state. Note that this does not mean that the corresponding amount of water masses crosses the isopycnal surfaces as mentioned above. Only one case is shown in Fig. 5 but such flattening of isopycnal surfaces is also found in all other cases. 


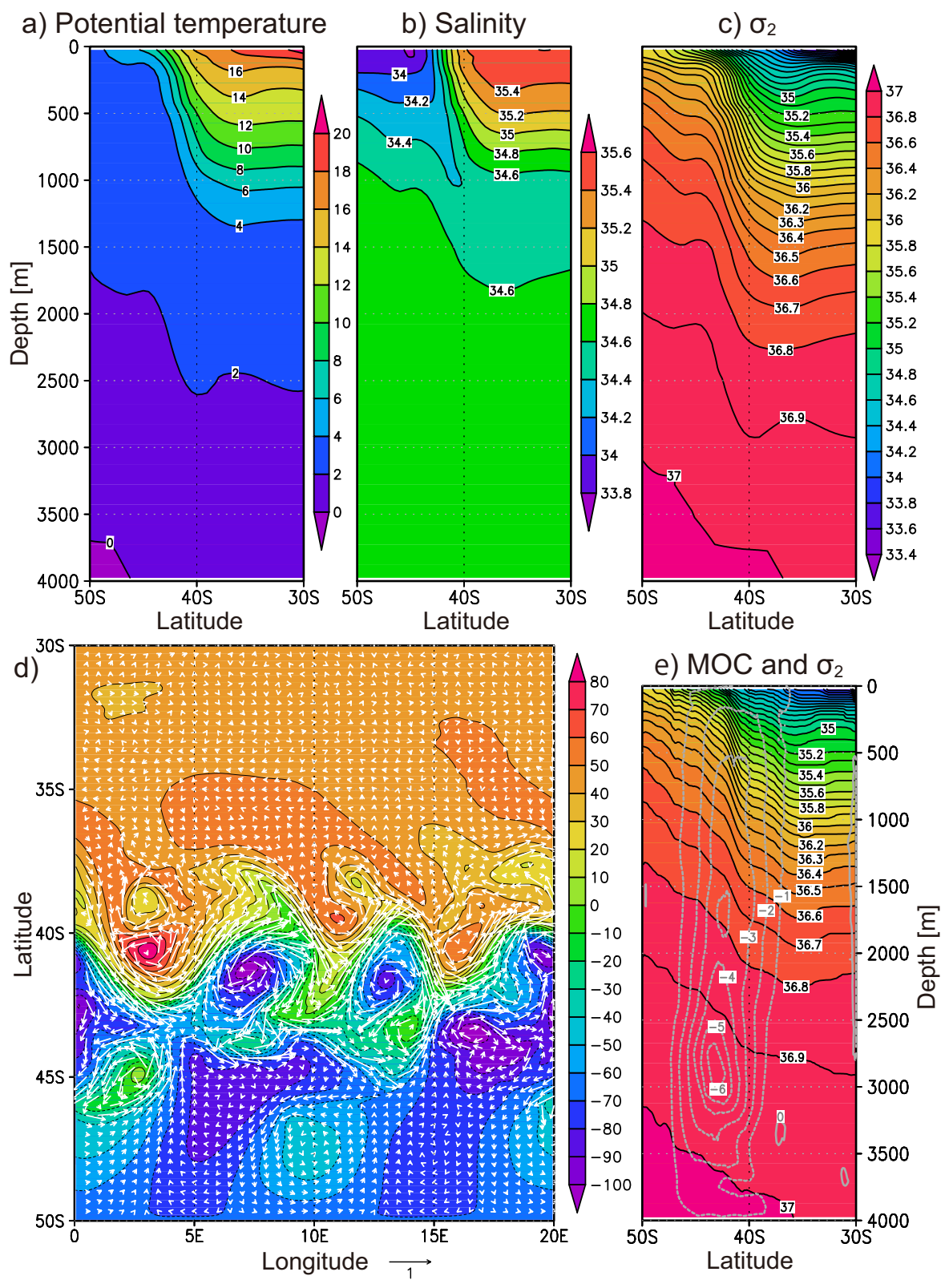

Figure 5: Initial conditions for (a) potential temperature, (b) salinity and (c) $\sigma_{2}$ for channel experiments. The lower left panel (d) shows a snapshot of the experiment with a nonlinear EOS and without explicit horizontal diffusion and external forcings (one of short-run experiments) at 180 day. The shade denotes the sea surface height and arrows denote the horizontal velocity of the top layer. The lower right panel (e) shows the zonal average of density at the final state and thęnnual average of meridional streamfunction (gray line). 


\subsection{Water mass transformation induced by numerical diffusion}

Here, we show water mass transformation rates in the short-run experiments. Meridional velocities are zero at the northern and southern solid boundaries. A meridional streamfunction becomes zero at $y=30^{\circ} \mathrm{S}$. The water mass transformation rate due to numerical diffusion $\left(\Omega_{D N}\right)$ there can be diagnosed with the following equation.

$$
\begin{aligned}
\Omega_{D N} & =\frac{\partial V}{\partial t}-\Omega_{D E}-\Omega_{C E}-\Omega_{C N}-\Omega_{Q} \\
& =\frac{\partial V}{\partial t}-\Omega^{\prime}
\end{aligned}
$$

where $\Omega_{Q}$ denotes the transformation rate due to convective adjustment, which is almost equal to zero.

The volume inflation term $(\partial V / \partial t)$ is comparable in magnitude to the sum of water mass transformation terms on the right hand of Eq. $12\left(\Omega^{\prime}\right)$ for $36.4<\sigma_{2}<36.9$ in the experiment without explicit horizontal diffusion (Fig. $6 a)$. It means that $\Omega_{D N}$, which is calculated as their difference, is almost zero there. Two vertical lines in Fig. 6 describe densities at 1000 and $3000 \mathrm{~m}$ depth in the virtual state where all isopycnal surfaces are adiabatically flattened, respectively. We define the region between these two lines as interior region. This result shows that numerical diffusion associated with the SOM scheme works little in the most part of the interior region compared with in the upper and lower boundary regions. Large numerical diffusion near the sea surface would result from the strong activity of mesoscale eddies. Numerical diffusion is also strong in the high density range $\left(\sigma_{2}>36.9\right)$, which corresponds to the region around the sea bottom.

Comparison of $\Omega_{D N}$ among the experiments with different diffusivity 

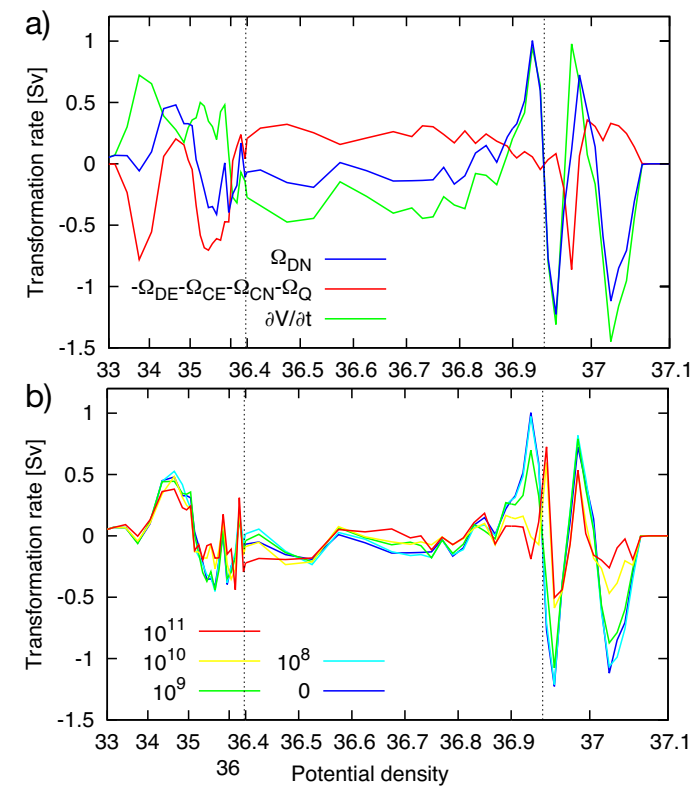

Figure 6: (a) Annual averages of the volume inflation term (green) and the water mass transformation rates due to explicit diffusion, explicit cabbeling, numerical cabbeling and convective adjustment (red) and numerical diffusion (blue) in the experiment with a nonlinear EOS and without explicit horizontal diffusion. (b) The water mass transformation rate due to numerical diffusion. Five colored lines denote the experiments with a horizontal diffusion coefficient of $10^{8}$ (cyan), $10^{9}$ (green), $10^{10}$ (yellow) and $10^{11} \mathrm{~m}^{4} \mathrm{~s}^{-1}$ (red) and the experiment without explicit horizontal diffusion (blue), respectively. Positive value denotes transformation toward the denser water mass. Two vertical dashed lines denote densities at 1000 and $3000 \mathrm{~m}$ depth in the state where all density surfaces are adiabatically flattened, respectively. 
shows that a larger diffusion coefficient leads to less numerical diffusion (Fig. $6 \mathrm{~b})$. This is remarkable in the high density range. Numerical diffusion is especially large in the three experiments with lower diffusivity in Fig. 6b and its magnitude is almost the same among them. It becomes significantly smaller with higher diffusivity $\left(10^{10}\right.$ and $\left.10^{11} \mathrm{~m}^{4} \mathrm{~s}^{-1}\right)$. Explicit diffusivity in the experiment LOW belongs to the former group and that in the experiment HIGH belongs to the latter. The results of the channel experiments are consistent with those of the Southern Ocean model. The volume inflation term $(\partial V / \partial t)$ differs little among these five experiments. This means that the change of $\Omega^{\prime}\left(=\Omega_{D E}+\Omega_{C E}+\Omega_{C N}+\Omega_{Q}\right)$ corresponds to the reduction of $\Omega_{D N}$ with higher diffusivity. The change of $\Omega_{D E}$ dominates that of $\Omega^{\prime}$ (not shown). Higher explicit diffusivity leads to larger $\Omega_{D E}$ which corresponds to smaller $\Omega_{D N}$.

These analyses are conducted based on the density $\sigma_{2}$. The use of single density more or less accompanies analytical errors (Iudicone et al., 2008). The conclusion should be validated by the experiments with a linear EOS. The extra sensitivity study is conducted with the following EOS:

$$
\sigma_{L}=\rho-1000=-0.2 \theta+0.8 s \text {. }
$$

The pattern of density distribution at the initial and final states is similar to that of Fig. 5c and e but the magnitude is smaller by around $9.5 \mathrm{~kg} \mathrm{~m}^{-3}$ below the $1000 \mathrm{~m}$ depth. Errors in diagnosing the numerical cabbeling effect can be excluded in these experiments. The water mass transformation due 
to numerical diffusion is calculated with the following equation.

$$
\begin{aligned}
\Omega_{D N} & =\frac{\partial V}{\partial t}-\Omega_{D E}-\Omega_{Q} \\
& =\frac{\partial V}{\partial t}-\Omega^{\prime}
\end{aligned}
$$

where $\Omega^{\prime}=\Omega_{D E}+\Omega_{Q}$ in the linear experiments.

Figure 7a shows $\partial V / \partial t, \Omega^{\prime}$ and $\Omega_{D N}$ in the experiment without explicit horizontal diffusion. The volume inflation term and $\Omega^{\prime}$ are almost the same in magnitude in the most part of the ocean interior, which means small numerical diffusion. $\Omega_{D N}$ contributes a lot to water mass transformation in both boundary regions and its magnitude decreases with increase in an explicit horizontal diffusion coefficient especially near the ocean bottom (Fig. 7b). By contrast, explicit diffusion becomes larger with increase in explicit horizontal diffusivity (Fig. 7c). These five experiments are also divided into two groups: high diffusivity group $\left(10^{10}, 10^{11} \mathrm{~m}^{4} \mathrm{~s}^{-1}\right)$ in which explicit diffusion dominates and low diffusivity group $\left(0,10^{8}, 10^{9} \mathrm{~m}^{4} \mathrm{~s}^{-1}\right)$ where numerical diffusion dominates.

Figure 8 shows absolute values of $\Omega_{D E}$ and $\Omega_{D N}$ at $\sigma_{L}=27.725$, where the transformation rates due to explicit and numerical diffusions most strongly appear, plotted against the explicit horizontal diffusivity $(\kappa) .\left|\Omega_{D N}\right|$ amounts to about $1.1 \mathrm{~Sv}$ in the experiment without explicit horizontal diffusion. Its magnitude varies little among the experiments with low diffusivity $\left(\kappa \leq 10^{9}\right)$ and decreases with rise of explicit diffusivity. Of course, $\left|\Omega_{D E}\right|$ increases with the explicit diffusivity. The vertical component accounts for about $0.3 \mathrm{~Sv}$ of $\left|\Omega_{D E}\right|$ and differs little among the all experiments. The horizontal component is very small or even almost zero in the low diffusivity experiments $\left(\kappa \leq 10^{9}\right)$. 

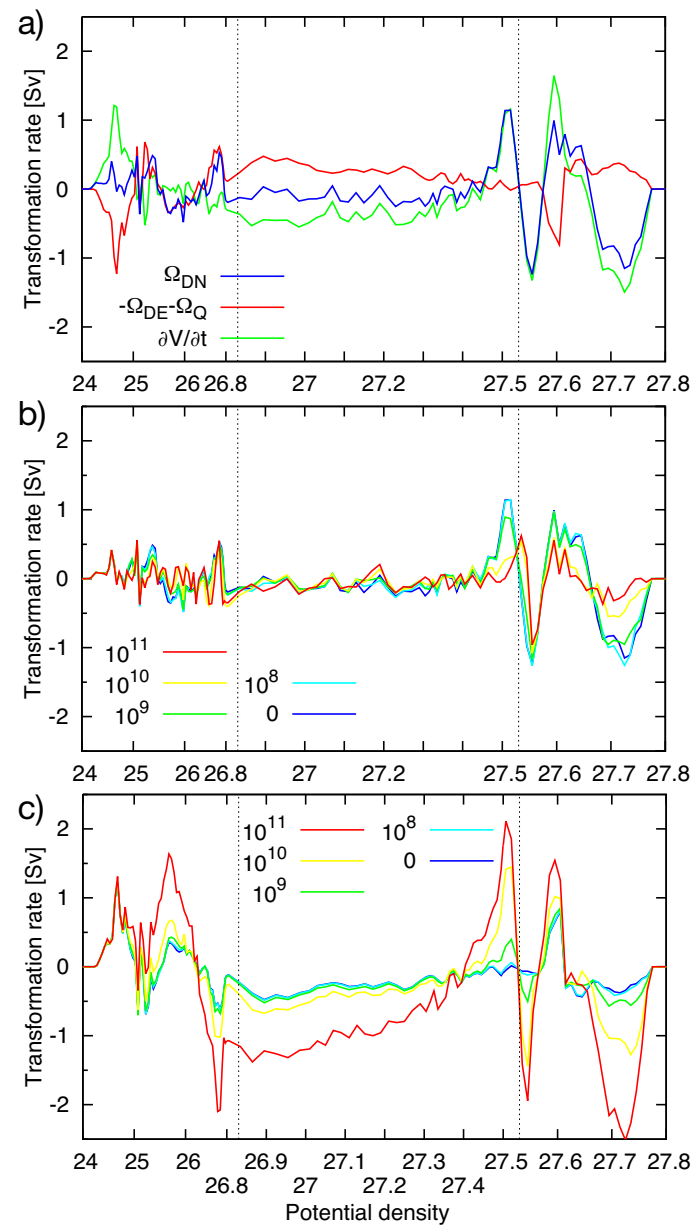

Figure 7: $(a, b)$ The same as Fig. 6 but in the experiments with a linear EOS and without explicit horizontal diffusion. (c) The same as Fig. $6 \mathrm{~b}$ but the water mass transformation rates induced by explicit diffusion in the experiments with a linear EOS. 


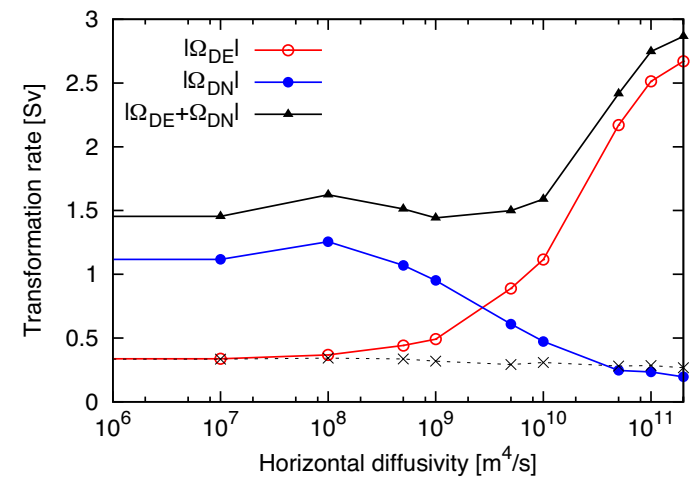

Figure 8: Absolute values of water mass transformation rates induced by explicit diffusion (red), numerical diffusion (blue) and total diffusions (both explicit and numerical diffusions; black solid) across the isopycnal surface $27.725 \sigma_{L}$. The black dashed line denotes the vertical component of $\Omega_{D E}$. The absolute value of $\Omega_{D N}$ is about $1.1 \mathrm{~Sv}$ in the experiment without horizontal diffusion.

Explicit diffusion dominates when $\kappa$ exceeds $10^{10} \mathrm{~m}^{4} \mathrm{~s}^{-1}$.

There seems to be three regimes as for the effect of diffusions. One is a "numerical diffusion" regime where the explicit horizontal diffusion scarcely works and the numerical diffusion dominantly accounts for water mass transformation due to diffusion. The second is a "transitional" regime where both the explicit and numerical diffusions comparably contribute. The last one is an "explicit diffusion" regime in which the explicit diffusion dominates in water mass transformation due to diffusion. Boundaries among them exist around $\kappa=10^{9}$ and $10^{10}$ for the isopycnal surface of $27.725 \sigma_{L}$, respectively. The experiment LOW with the Southern Ocean model belongs to the numerical diffusion regime and the experiment HIGH falls between the transitional and explicit diffusion regimes. This classification into three regimes are consistent with the results of the Southern Ocean model that numerical diffusion 


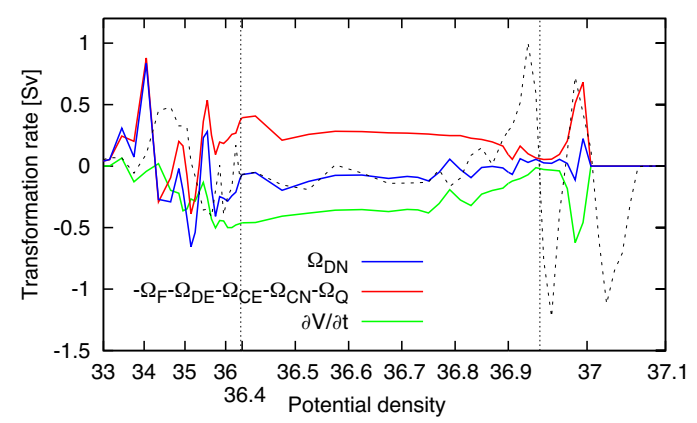

Figure 9: The same as Fig. 6a but in the long-run experiment. The black dashed denotes $\Omega_{D N}$ for the experiment shown in Fig. 6a (the blue line there).

dominates in the experiment LOW and explicit diffusion dominates in the experiment HIGH.

\subsection{Deep stratification and numerical diffusion}

Hill et al. (2012) also conduct channel experiments under an idealized configuration with the use of SOM scheme and find that numerical diffusion is negligibly small in the interior region. They also find that numerical diffusion is strong near the boundaries. This study is consistent with their study. On the other hand, the diffusivity near the abyss is obviously smaller than that near the sea surface in their study (see their Fig. 4). In contrast, $\Omega_{D N}$ in this study is larger near the sea bottom than near the sea surface. Water mass transformation induced by diffusions depends on diffusivity and spatial gradients of density. Because stratification is clearly weaker near the abyss, it is supposed that the diffusivity of numerical diffusion near the sea bottom is much larger than that near the sea surface in this study.

This strong numerical diffusion near the abyss plays an important role in simulating the Southern Ocean as already discussed and deserves further 
investigations. The difference between these two studies might result from a difference of the integration period. Hill et al. (2012) drive their model with external forcings until the model is spun up to statistical equilibrium. So, we drive our channel model for 40 years with surface winds and buoyancy fluxes and analyze the last 1 year. The explicit horizontal diffusivity is set to zero. $\Omega_{D N}$ in the interior region is small as well as that in the shortrun experiment (blue and black dashed lines in Fig. 9, respectively). It is comparable or even larger than that in the short-run experiment near the sea surface. This is because thermal and haline fronts near the surface are maintained by damping sea surface temperature and salinity to climatology and this leads to a stronger activity of eddies. On the other hand, $\Omega_{D N}$ near the ocean bottom significantly decreases in the long-run experiment. Its magnitude is comparable with that in the interior.

The magnitude of numerical diffusion depends on velocity and the spacial gradient of the tracer. Figure 10a shows annual averages of horizontally integrated mean and eddy kinetic energy (MKE and EKE) of the long-run experiment and the short-run experiment without explicit horizontal diffusion. Because the wind stress imposed in the long-run experiment supplies a large amount of kinetic energy, both MKE and EKE exceed those in the short-run experiment, respectively. However, this does not necessarily mean larger numerical diffusion in the long-run experiment than in the short-run experiment. For example, uniform tracer distributions will result in zero $\Omega_{D N}$. In fact, there are no water masses denser than $37 \sigma_{2}$ in the long-run experiment (Fig. 9). Such dense water masses are not produced in this area (at $49^{\circ} \mathrm{E}$ and from $50^{\circ} \mathrm{S}$ to $30^{\circ} \mathrm{S}$ ). They are modified into lighter water masses 
by strong numerical diffusion near the bottom and disappears after the long integration period.

The strong numerical diffusion also results in smoother gradients of potential temperature and salinity near the bottom (above the isopycnal surface of $37 \sigma_{2}$ ) in the long-run experiment. The horizontal density gradient there is smaller than that in the short-run experiment by a factor of about 5 (Fig. 10b). It is also smaller than the density gradient in the experiment LOW which also suffers from large numerical diffusion near the bottom. The deep stratification is an important key of the numerical diffusion around the abyss. The dense bottom water formation at high latitudes leads to density gradients near the bottom in the real ocean. The lack of this bottom water supply results in weak deep stratification and small numerical diffusion in the long-run experiment. Although the experiment LOW and the shortrun experiment also have the problem of insufficient dense water formation, they retain realistic distributions of potential temperature and salinity due to their short integration period. The density gradient in the short-run experiment is close to that in the experiment LOW around $4000 \mathrm{~m}$ depth. We believe that the short-run experiment better reproduces what occurs near the bottom in the real ocean than our long-run experiment. As already discussed, applying high explicit diffusivity for less numerical diffusion leads to unrealistically diffusive deep stratification. These results indicate that numerical diffusion must be significantly large near the bottom as long as the near bottom stratification is realistically simulated. 

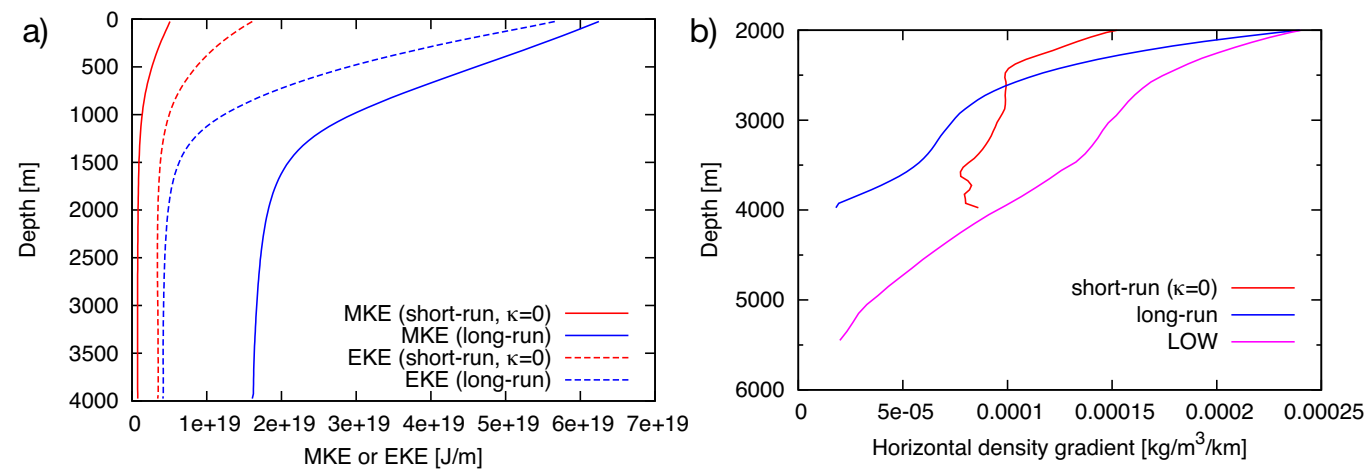

Figure 10: (a) Annual averages of horizontally integrated MKE (solid) and EKE (dashed) in the long-run experiment (blue) and the short-run experiment without explicit horizontal diffusion (red). (b) Absolute values of the horizontal $\sigma_{2}$ gradient $\left(\overline{\left|\nabla_{h} \sigma_{2}\right|}\right)$ in the short-run experiment without explicit horizontal diffusivity (red), the long-run experiment (blue) and the experiment LOW (purple). They are annually and horizontally averaged. The gradient in the experiment LOW is calculated with data in the Southern Ocean (south to $\left.30^{\circ} \mathrm{S}\right)$. Unit is $\mathrm{kg} \mathrm{m}^{-3} \mathrm{~km}^{-1}$.

\section{Conclusions and discussions}

This study investigates the effect of numerical diffusion from the viewpoint of water mass transformation. We conduct a set of idealized experiments with a channel model. Numerical integration starts from zonally uniform distributions of potential temperature and salinity, which are taken from WGHC at $49^{\circ} \mathrm{E}$ and from $50^{\circ} \mathrm{S}$ to $30^{\circ} \mathrm{S}$. The model is run for 1 year without external forcings. These experiments show that numerical diffusion is small in the ocean interior region. On the other hand, the experiment without explicit diffusion shows that numerical diffusion is significantly large near the sea surface and especially around the sea bottom. Because of its short integration period, the model retains strong initial stratification near 
the abyss, which leads to large water mass transformation due to numerical diffusion there. Such a situation has not been focused on in earlier studies.

The sensitivity study against the explicit horizontal diffusivity shows that the magnitude of these numerical diffusion effects tends to become small with increase in the explicit diffusivity. Of course, explicit diffusion tends to be strong with increase in the explicit diffusivity. There are three regimes as to the effects of diffusions. One is the numerical diffusion regime, where explicit diffusion hardly works. Numerical diffusion associated with an advection scheme dominantly diffuses fine structures created by mesoscale eddies there, and its magnitude varies little with explicit horizontal diffusivity. So, the water mass transformation rate due to total diffusion remains almost constant there. Another regime is the transitional regime, where both explicit and numerical diffusions are important. Increase in explicit horizontal diffusivity leads to increase (decrease) in the explicit (numerical) diffusion there. The last regime is the explicit diffusion regime. The total diffusion effect there becomes significantly larger than those in the other two regimes. Explicit diffusion dominates in this total diffusion effect and numerical diffusion is almost negligible there. Some readers might doubt that the small effect of numerical diffusion results from cancellation between positive and negative water mass transformation rates induced by numerical diffusion because it is not sign-definite. This study also investigates a sensitivity of the cabbeling effect, which is positive definite ${ }^{6}$, on explicit horizontal diffusivity (Appendix

\footnotetext{
${ }^{6}$ Strictly speaking, the cabbeling effect induced by the biharmonic diffusion is not necessarily positive definite (Delhez \& Deleersnijder, 2007). As already noted in the previous footnote, however, it is confirmed that the effect of such an anti-diffusive process is small
} 
C). It shows consistent results with those noted above and suggests that the small numerical diffusion does not result from cancellation between positive and negative transformation rates.

The Southern Ocean model would not suffer from numerical diffusion if we use an explicit horizontal diffusion coefficient which belongs to the explicit diffusion regime. However, our two experiments under the realistic configuration show that it is not a better option for the Southern Ocean modeling. The two experiments, LOW and HIGH, belong to the numerical diffusion and the transitional regimes, respectively. Results of both experiments are consistent with those of channel experiments. That is, numerical diffusion dominates in the experiment LOW and works little in the experiment HIGH. The pattern of the water mass transformation due to the total diffusion resembles each other, which indicates that the numerical diffusion well duplicates the explicit diffusion. The total diffusion is especially large in the high density range, which corresponds to the region near the bottom, in both experiments. This leads to reduction in the volume of AABW by transforming it to the lighter water mass. Although inadequate DSW formation around Antarctica is mainly blamed for this AABW loss, UH discuss that the water mass transformation due to the total diffusion might be too diffusive in the experiment HIGH for a realistic simulation of the Southern Ocean. It is naturally expected that diffusivity in the explicit diffusion regime would also be too diffusive for our eddy-resolving model of the Southern Ocean.

We cannot find the most adequate diffusivity for the Southern Ocean

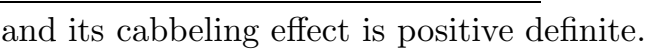


models with horizontal resolution around 0.1 degree from the results of the current study. However, it seems robust that an explicit diffusion coefficient in the explicit diffusion regime is inadequate for a realistic simulation of the Southern Ocean with such models. This indicates that such models are not free from numerical diffusion associated with advection schemes even if the models adopt high-accuracy advection schemes such as the SOM scheme. So, we must adequately diagnose its effect when analyzing outputs of such high-resolution models. The current study proposes a method to evaluate numerical diffusion in terms of water mass transformation, which has been used in this study to reach the conclusion noted above. Water mass transformation due to numerical diffusion (including cabbeling) can be evaluated as the difference between the net water mass transformation $(=\psi+\partial V / \partial t)$ and the explicit water mass transformation which can be directly calculated $\left(\Omega_{D E}+\Omega_{C E}+\Omega_{Q}\right.$ in Eq. 10). Numerical cabbeling $\left(\Omega_{C N}\right)$ can be estimated with the use of the method proposed by UH (also shown in Appendix A). Subtracting $\Omega_{C N}$ from the numerical diffusion effect calculated above, we can get the water mass transformation due to numerical diffusion of density $\left(\Omega_{D N}\right)$. This method is a useful tool for investigating an eddying ocean with a numerical model.

Ilıcak et al. (2012) show that applying high viscosity significantly reduces numerical diffusion. Of course, it is desirable to reduce its effect because it is difficult to analyze as is easily seen from the discussion above. However, high viscosity leads to reduction of eddy kinetic energy (EKE). Thoppil et al. (2011) show that EKE in a model with horizontal resolution of $1 / 12.5^{\circ}$ is deficient and accounts for only about $80 \%$ of observational estimates. So, 
simply applying high viscosity might not be an appropriate option for a realistic simulation of the ocean.

Thoppil et al. (2011) also find that EKE increases to the level consistent with observation by doubling horizontal resolution. Numerical diffusion must have a direct relationship with other parameters such as grid spacing and time step sizes along with scales of velocities and tracer gradients that are discussed in the current study. However, it is doubtful that such finer resolution could lead to reduction of numerical diffusion. Higher resolution results in representing finer distributions of tracers and more rapid velocity fields, which lead to stronger numerical diffusion. If the model's resolution is high enough that resolved velocity and length scales do not differ from those of sub-grid scale processes, increase of resolution would lead to reduction of numerical diffusion. Such resolution will be at least finer than sub-mesoscale. It will be important in the regime of models with such finer resolutions to pursue the relationship between numerical diffusion and grid spacing and time step sizes, which cannot be found in the current study because of single horizontal resolution and time step size of the experiments. For the time being, however, such a high resolution modeling seems not to be feasible for studies of the ocean general circulation. So, we need to properly evaluate the numerical diffusion when analyzing outputs of high resolution models.

\section{Acknowledgment}

The authors thank to Drs. A. Oka, Y. Komuro, H. Tatebe, M. Kurogi, M. Watanabe, K. Kusahara, Y, Sasajima, Y. Matsumura, T. Kawasaki and Y. Hiraike for their helpful comments and discussions. This study is supported 
by Japan Science and Technology Agency/Core Research for Evolutional Science and Technology, Research Fellowships of the Japan Society for the Promotion of Science for Young Scientists, and Initiative on Promotion of Supercomputing for Young Researchers, Supercomputing Division, Information Technology Center, The University of Tokyo. Numerical calculations were done by HA8000 and SR16000 at Information Technology Center, University of Tokyo. Figures in this paper are produced by Grid Analysis and Display System and gnuplot. Our thanks to the editors and four anonymous reviewers, whose comments helped to improve the draft of this paper.

\section{Appendix A. A method for diagnosing the effect of numerical cabbeling}

The advection-diffusion equations for potential temperature and salinity in a model are described as:

$$
\begin{aligned}
& \frac{\partial \theta}{\partial t}=-\mathcal{A}(\theta)+\mathcal{D}(\theta), \\
& \frac{\partial s}{\partial t}=-\mathcal{A}(s)+\mathcal{D}(s),
\end{aligned}
$$

where $\mathcal{A}(\phi)$ is the advection term for an arbitrary tracer $\phi$ in a model, and the source/sink terms of potential temperature and salinity $\left(q_{\theta}\right.$ and $q_{s}$ in Eqs. 4 and 5 are neglected). This term can be decomposed into the contributions from true advection and numerical diffusion ${ }^{7}$ as:

$$
-\mathcal{A}(\phi)=-\mathbf{u} \cdot \nabla \phi+\nabla \cdot \mathbf{F}(\phi)
$$

\footnotetext{
${ }^{7}$ The term "numerical errors" might be more appropriate for this effect but the word "numerical diffusion" is used here and also in the main text.
} 
where $\mathbf{F}(\phi)$ denotes the flux of $\phi$ due to numerical diffusion. The contribution of these advection terms to the tendency of density can be described as:

$$
\begin{aligned}
-\alpha^{*} \mathcal{A}(\theta)-\beta^{*} \mathcal{A}(s) & =-\mathbf{u} \cdot\left(\alpha^{*} \nabla \theta+\beta^{*} \nabla s\right)+\alpha^{*} \nabla \cdot \mathbf{F}(\theta)+\beta^{*} \nabla \cdot \mathbf{F}(s) \\
& =-\mathbf{u} \cdot \nabla \sigma+\alpha^{*} \nabla \cdot \mathbf{F}(\theta)+\beta^{*} \nabla \cdot \mathbf{F}(s) .
\end{aligned}
$$

The last two terms in the second line can be decomposed into the density diffusion term $\left(\nabla \cdot \mathbf{F}^{\prime}(\sigma)\right)$ and the cabbeling term $\left(\mathcal{C}_{\text {num }}\right)$ as in the case of the physical diffusion.

$$
\begin{aligned}
\alpha^{*} \nabla \cdot \mathbf{F}(\theta)+\beta^{*} \nabla \cdot \mathbf{F}(s) & =\nabla \cdot\left[\alpha^{*} \mathbf{F}(\theta)+\beta^{*} \mathbf{F}(s)\right]-\left[\nabla \alpha^{*} \cdot \mathbf{F}(\theta)+\nabla \beta^{*} \cdot \mathbf{F}(s)\right] \\
& =\nabla \cdot \mathbf{F}^{\prime}(\sigma)+\mathcal{C}_{n u m}
\end{aligned}
$$

Here, we describe the sum of true advection and numerical diffusion terms of density as follows:

$$
-\mathbf{u} \cdot \nabla \sigma+\nabla \cdot \mathbf{F}^{\prime}(\sigma)=-\mathcal{A}^{\prime}(\sigma)
$$

The advection-diffusion equation for density can be rewritten as:

$$
\begin{aligned}
\frac{\partial \sigma}{\partial t} & =-\alpha^{*} \mathcal{A}(\theta)-\beta^{*} \mathcal{A}(s)+\mathcal{D}(\sigma)+\mathcal{C} \\
& =-\mathcal{A}^{\prime}(\sigma)+\mathcal{C}_{\text {num }}+\mathcal{D}(\sigma)+\mathcal{C}
\end{aligned}
$$

Therefore, the cabbeling effect associated with the numerical diffusion can be diagnosed by:

$$
\mathcal{C}_{\text {num }}=\mathcal{A}^{\prime}(\sigma)-\left[\alpha^{*} \mathcal{A}(\theta)+\beta^{*} \mathcal{A}(s)\right]
$$

By substituting this term in Eq. 10, we can diagnose the cabbeling effect on the water mass transformation. 
The way to evaluate the advection term of density $\mathcal{A}^{\prime}(\sigma)$ has not been clarified. We adopt a way to calculate advected density $\left(\sigma_{a}=\sigma\left(\theta_{a}, s_{a}\right)\right)$ by substituting advected potential temperature and salinity $\left(\theta_{a}\right.$ and $\left.s_{a}\right)$ into the EOS. Here, we describe the true advected potential temperature and salinity as $\theta_{t}$ and $s_{t}$. There holds the following relationships between $\theta_{a}$ and $\theta_{t}$, and $s_{a}$ and $s_{t}$ :

$$
\begin{aligned}
& \theta_{a}=\theta_{t}+\delta \theta, \\
& s_{a}=s_{t}+\delta s,
\end{aligned}
$$

where $\delta \theta$ and $\delta s$ denote differences between the two advected variables. Note that terms in the right hand sides of these equations are all unknown. We can describe the tracer flux due to numerical diffusion in the Eq. A.3 as follows.

$$
\begin{aligned}
& \mathbf{F}(\theta)=-\mathbf{u} \cdot \delta \theta, \\
& \mathbf{F}(s)=-\mathbf{u} \cdot \delta s .
\end{aligned}
$$

Then, $\mathcal{A}^{\prime}(\sigma)$ in Eq. A.6 can be described as follows.

$$
\begin{aligned}
\mathcal{A}^{\prime}(\sigma) & =\mathbf{u} \cdot \nabla \sigma_{t}-\nabla \cdot\left[\alpha^{*} \mathbf{F}(\theta)+\beta^{*} \mathbf{F}(s)\right] \\
& =\mathbf{u} \cdot \nabla\left(\sigma_{t}+\alpha^{*} \delta \theta+\beta^{*} \delta s\right) .
\end{aligned}
$$

This equation contains three unknown variables $\left(\sigma_{t}, \delta \theta\right.$ and $\left.\delta s\right)$. On the other hand, the Taylor series of the true advected density $\left(\sigma_{t}=\sigma\left(\theta_{t}, s_{t}\right)\right)$ around $\left(\sigma_{a}, s_{a}\right)$ is

$$
\sigma_{t}=\sigma_{a}-\alpha^{*}\left(\theta_{a}, s_{a}\right) \delta \theta-\beta^{*}\left(\theta_{a}, s_{a}\right) \delta s+\mathcal{O}\left(\delta^{2}\right),
$$


where $\delta$ denotes a scale of $\delta \theta$ and $\delta s$. We can approximately evaluate $\mathcal{A}^{\prime}(\sigma)$ with the errors that are quadratic in the scale $\delta$ as follows.

$$
\mathcal{A}^{\prime}(\sigma) \sim \mathbf{u} \cdot \nabla \sigma_{a}
$$

Note that we assume that the difference between $\alpha^{*}\left(\beta^{*}\right)$ in Eq. A.13 and $\alpha^{*}\left(\theta_{a}, s_{a}\right)\left(\beta^{*}\left(\theta_{a}, s_{a}\right)\right)$ is negligible.

\section{Appendix B. Discrete representations}

Figure B.11 shows a schematic of the model grid system. A black point denotes a tracer point. The number of grids in the $\mathrm{x}, \mathrm{y}$ and $\mathrm{z}$ directions are $N X, N Y$ and $N Z$, respectively. The volume inflation term $(\partial V / \partial t)$ is calculated from profiles of potential temperature and salinity at the initial $(t=0)$ and final $(t=T)$ states. A volume of each grid is defined as $v_{i, j, k} \equiv$ $\Delta x \Delta y \Delta z$. We consider $N$ isopycnal layers whose boundaries are described as $\sigma_{n}(0 \leq n \leq N)$. We can calculate the volume below the isopycnal surface $S_{\sigma_{n}}$, whose density is $\sigma_{n}$, and from the southern boundary of the model to the grid of $j=n y$ as follows.

$$
V_{n y+\frac{1}{2}, n, t}=\sum_{k=1}^{N Z} \sum_{j=1}^{n y} \sum_{i=1}^{N X} v_{i, j, k} U\left(\sigma_{i, j, k, t}-\sigma_{n}\right),
$$

where

$$
U(x)= \begin{cases}1 & \text { if } x>0, \\ 0 & \text { if } x \leq 0 .\end{cases}
$$

The volume inflation term is diagnosed as

$$
\left.\frac{\partial V}{\partial t}\right|_{n y+\frac{1}{2}, n}=\frac{V_{n y+\frac{1}{2}, n, T}-V_{n y+\frac{1}{2}, n, 0}}{T} .
$$




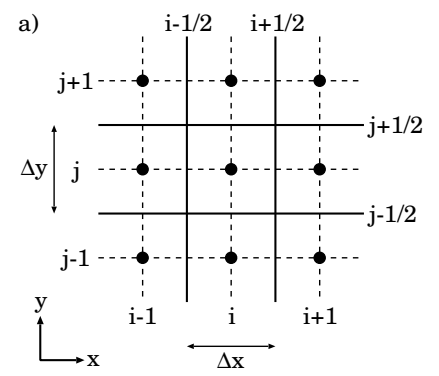

b)

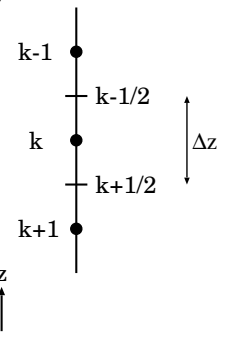

Figure B.11: A schematics for model grids.

$\psi$ is defined as an annual average of meridional streamfunctions at all snapshots. It is calculated as follows.

$$
\psi_{n y+\frac{1}{2}, n}=-\frac{1}{N T} \sum_{m=1}^{N T} \sum_{k=1}^{N Z} \sum_{j=1}^{n y} \sum_{i=1}^{N X} F_{i, j+\frac{1}{2}, k, t_{m}}^{v} U\left(\sigma_{i, j+\frac{1}{2}, k, t_{m}}-\sigma_{n}\right),
$$

where $N T$ is the number of snapshots, $F_{i, j+\frac{1}{2}, k, t_{m}}^{v}$ is the northward volume transport across the northern boundary of the grid $(i, j, k)$ at $t=t_{m}$ and $\sigma_{i, j+\frac{1}{2}, k, t_{m}}=\left(\sigma_{i, j, k, t_{m}}+\sigma_{i, j+1, k, t_{m}}\right) / 2$.

$\Omega$ is also calculated as an annual average of water mass transformation rates at all snapshots. For the ease of introduction, we consider an advectiondiffusion equation of density without numerical diffusion, cabbeling and other source/sink terms. There remains a convergence/divergence term of explicit diffusion fluxes across the lateral boundaries of the grid box, $\mathcal{D}_{i, j, k, t_{m}}(\sigma)$. We can calculate the water mass transformation rate induced by explicit diffusion with the use of Eq. 10. The volume integration part is described as follows.

$$
G_{n y+\frac{1}{2}, n, t_{m}}=\sum_{k=1}^{\mathrm{NZ}} \sum_{j=1}^{n y} \sum_{i=1}^{\mathrm{NX}} \mathcal{D}_{i, j, k, t_{m}}(\sigma) U\left(\sigma_{n}-\sigma_{i, j, k, t_{m}}\right) .
$$

So, the water mass transformation rate can be calculated as

$$
\Omega_{n y+\frac{1}{2}, n+\frac{1}{2}}=\frac{1}{N T} \sum_{m=1}^{\mathrm{NT}} \frac{G_{n y+\frac{1}{2}, n+1, t_{m}}-G_{n y+\frac{1}{2}, n, t_{m}}}{\sigma_{n+1}-\sigma_{n}} .
$$


Since the water mass transformation rate is defined on different isopycnal surfaces from the volume inflation term and meridional streamfunction, the latter two need to be interpolated on the middle of the isopycnal surfaces on which the volume inflation term and the meridional streamfunction are calculated.

Each grid box belongs to only one density layer according to the density at its center in the current study. This is a simplest way to convert variables from a z-coordinate to a density-coordinate. Getzlaff et al. (2010) shortly discuss such a method of conversion and they adopt an improved method. We need to check whether evaluation of this term is sensitive to its method for calculation. Figure B.12 compares water mass transformation rates and $\partial V / \partial t$ calculated with the two methods. There is little difference between $\Omega_{D N}$ diagnosed with the use of two methods. The conclusion of the current study does not depend on the way to calculate water mass transformation rates, meridional overturning circulation and the volume inflation term.

\section{Appendix C. The cabbeling effect}

The cabbeling effect is another indicator of the magnitude of diffusions. The explicit cabbeling becomes larger and the numerical cabbeling becomes smaller with higher explicit diffusivity (Fig. C.13a and b). Figure C.13c shows the water mass transformation rates across the isopycnal surface 35.75 $\sigma_{2}$. The red and blue lines denote the effect of explicit cabbeling and numerical cabbeling ( $\Omega_{C E}$ and $\Omega_{C N}$ ), respectively. The black line shows the sum of both effects. The dashed line denotes the vertical component of $\Omega_{C E}$. The numerical diffusion, transitional and explicit diffusion regimes are clearly 

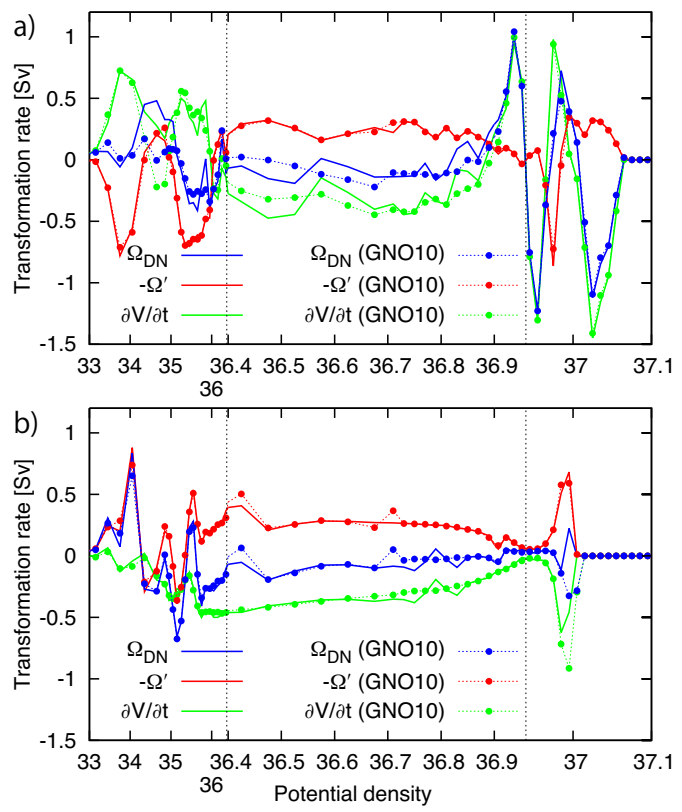

Figure B.12: The same as (a) Fig. 6a and (b) Fig. 9. $\Omega^{\prime}=\Omega_{F}+\Omega_{D E}+\Omega_{C E}+\Omega_{C N}+\Omega_{Q}$ $\left(\Omega_{F}=0\right.$ in (a)). $\Omega_{D N},-\Omega^{\prime}$ and $\partial V / \partial t$ calculated with the method proposed by Getzlaff et al. (2010) (GNO10) are also shown with doted blue, red and green lines with points, respectively. 
found there. Boundaries among these regimes are found around $10^{9}$ and $10^{10} \mathrm{~m}^{4} \mathrm{~s}^{-1}$, respectively. $\Omega_{C E}\left(\Omega_{C N}\right)$ becomes large (small) with increase in explicit horizontal diffusivity. $\Omega_{C N}$ differs little for $\kappa<10^{9}$. The same holds in almost all of other density surfaces as expected from Fig. C.13a and b.

\section{References}

Boyer, T. P., Stephens, C., Antonov, J. I., Conkright, M. E., Locarnini, R. A., O'Brien, T. D., \& Garcia, H. E. (2002). World Ocean Atlas 2001 Volume 2: Salinity. NOAA Atlas NESDIS, 50, 165pp.

Burchard, H., \& Rennau, H. (2008). Comparative quantification of physically and numerically induced mixing in ocean models. Ocean Modelling, 20, 293-311.

Delhez, E. J. M., \& Deleersnijder, E. (2007). Overshootings and spurious oscillations caused by biharmonic mixing. Ocean Modelling, 17, 183-198.

Getzlaff, J., Nurser, G., \& Oschlies, A. (2010). Diagnostics of diapycnal diffusivity in z-level ocean models part i: 1-dimensional case studies. Ocean Modelling, 35, 173-186.

Gouretski, V. V., \& Koltermann, K. P. (2004). WOCE Global Hydrographic Climatology. Technical Report 35/2004 Bundesamt für Seeschifffahrt und Hydrographie.

Griffies, S. M., \& Hallberg, R. W. (2000). Biharmonic friction with a Smagorinsky-like viscosity for use in large-scale eddy-permitting ocean models. Mon. Wea. Rev., 128, 2935-2946. 

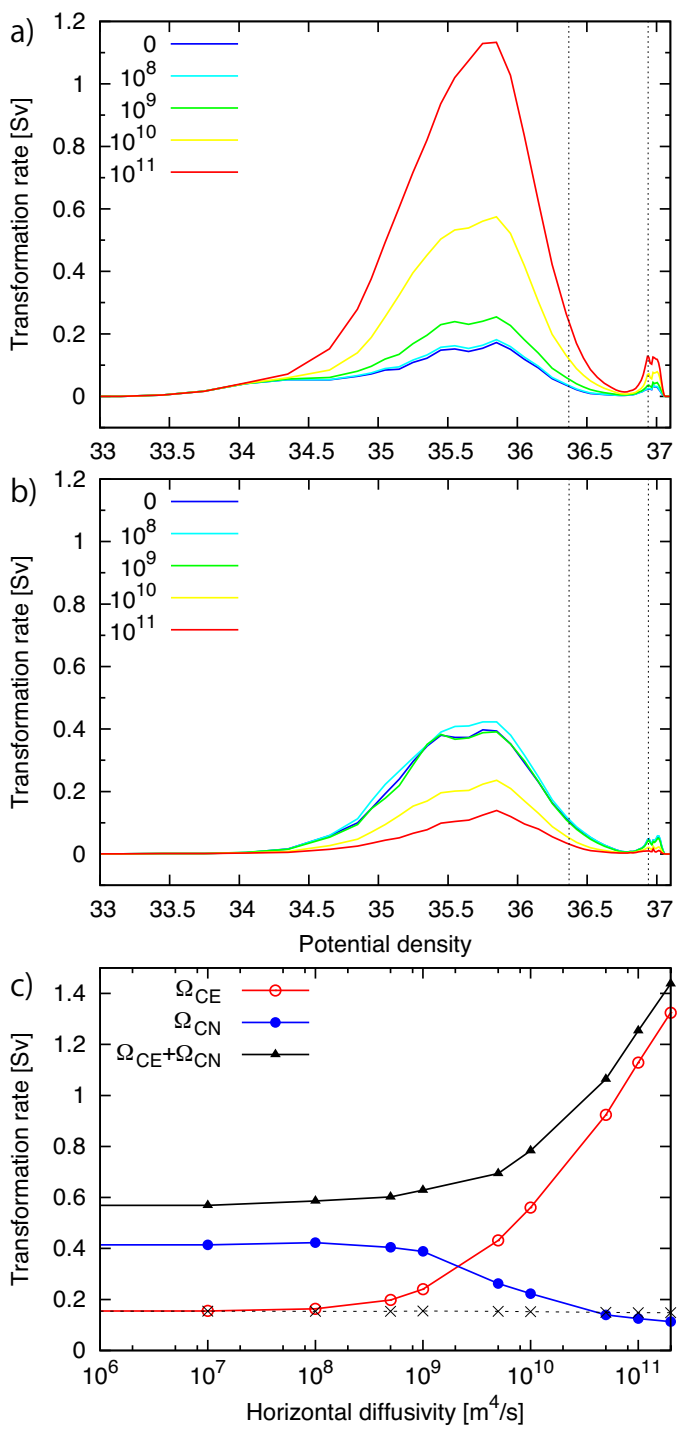

Figure C.13: (a, b) The same as Fig. $6 \mathrm{~b}$ but the water mass transformation rates due to cabbeling associated with the explicit diffusion $\left(\Omega_{C E}\right)$ and due to cabbeling associated with numerical diffusion $\left(\Omega_{C N}\right)$, respectively. Note that intervals for the horizontal axis are uniform here. The black dashed lines here are same as those in Fig. 6. (c) Values of $\Omega_{C E}$ (red) and $\Omega_{C N}$ (blue) at $35.75 \sigma_{2}$ in the channel experiments. Sum of them is described by a black solid line. The transformation rate due to numerical cabbeling is 0.4 $\mathrm{Sv}$ in the experiment with no explicit diffusion coefficient. A black dashed line shows the water mass transformation rate due to cabbeling induced by vertical diffusion. 
Griffies, S. M., Pacanowski, R. C., \& Hallberg, R. W. (2000). Spurious Diapycnal Mixing Associated with Advection in a z-Coordinate Ocean Model. Mon. Wea. Rev., 128, 538-564.

Haran, T. J., Bohlander, J., Scambos, T., Painter, T., \& Fahnestock, M. (2005). MODIS Mosaic of Antarctica (MOA) Image Map, http://nsidc.org/data/nsidc-0280.html. Digital Media.

Hasumi, H. (2006). CCSR Ocean Component Model (COCO) version 4.0. CCSR report 25 Center for Climate System Research, Univ. of Tokyo.

Hill, C., Ferreira, D., Campin, J.-M., Marshall, J., Abernathey, R., \& Barrier, N. (2012). Controlling spurious diapycnal mixing in eddy-resolving heightcoordinate ocean models — insights from virtual deliberate tracer release experiments. Ocean Modelling, 45-46, 14-26.

Ilıcak, M., Adcroft, A. J., Griffies, S. M., \& Hallberg, R. W. (2012). Spurious dianeutral mixing and the role of momentum closure. Ocean Modelling, $45-46,37-58$.

IOC, IHO, \& BODC (2003). Centenary Edition of the GEBCO Digital Atlas. published on CD-ROM on behalf of the Intergovernmental Oceanographic Commission and the International Hydrographic Organization as part of the General Bathymetric Chart of the Oceans.

Ishizaki, H., \& Motoi, T. (1999). Reevaluation of the Takano-Oonishi scheme for momentum advection on bottom relief in ocean models. J. Atmos. Oceanic Technol., 16, 1994-2010. 
Iudicone, D., Madec, G., \& McDougall, T. J. (2008). Water-mass transformation in a neutral density framework and the key role of light penetration. J. Phys. Oceanogr., 38, 1357-1376.

Kusahara, K., Hasumi, H., \& Tamura, T. (2010). Modeling sea ice production and dense shelf water formation in coastal polynyas around East Antarctica. J. Geophys. Res., 115, C10006.

Kusahara, K., Hasumi, H., \& Williams, G. D. (2011). Impact of the Mertz Glacier Tongue calving on dense shelf water formation and export. Nat. Commun., 2, doi: 10.1038/ncomms1156.

Lee, M.-M., Coward, A. C., \& Nurser, A. J. G. (2002). Spurious diapycnal mixing of the deep waters in an eddy-permitting global ocean model. $J$. Phys. Oceanogr., 32, 1522-1535.

Lee, M.-M., Nurser, A. J. G., Coward, A. C., \& de Cuevas, B. A. (2007). Eddy advective and diffusive transports of heat and salt in the Southern Ocean. J. Phys. Oceanogr., 37, 1376-1393.

Marsh, R., Nurser, A. J. G., Megann, A. P., \& New, A. L. (2000). Water mass transformation in the Southern Ocean of a global isopycnal coordinate GCM. J. Phys. Oceanogr., 30, 1013-1045.

Masumoto, Y., Sasaki, H., Kagimoto, T., Komori, N., Ishida, A., Sasai, Y., Miyama, T., Motoi, T., Mitsudera, H., Takahashi, K., Sakuma, H., \& Yamagata, T. (2004). A fifty-year eddy-resolving simulation of the world ocean - preliminary outcomes of OFES (OGCM for the Earth Simulator) -. Journal of the Earth Simulator, 1, 35-56. 
Morales Masqueda, M. A., \& Holloway, G. (2006). Second-order moment advection scheme applied to Arctic Ocean simulation. Ocean Modelling, $14,197-221$.

Prather, M. J. (1986). Numerical advection by conservation of second order moments. J. Geophys. Res., 91, 6671-6681.

Röske, F. (2001). An atlas of surface fluxes based on the ECMWF Re-Analysis - a climatological dataset to force global ocean general circulation models. MPI-Report 323 Max-Planck-Institut für Meteorologie.

Sloyan, B. M., \& Rintoul, S. R. (2001). The Southern Ocean limb of the global deep overturning circulation. J. Phys. Oceanogr., 31, 143-173.

Stephens, C., Antonov, J. I., Boyer, T. P., Conkright, M. E., Locarnini, R. A., O’Brien, T. D., \& Garcia, H. E. (2002). World ocean atlas 2001 volume 1: Temperature. NOAA Atlas NESDIS, 49, 167pp.

Tanaka, Y., \& Hasumi, H. (2008a). Injection of Antarctic Intermediate Water into the Atlantic subtropical gyre in an eddy resolving ocean model. Geophys. Res. Lett., 35, L11601.

Tanaka, Y., \& Hasumi, H. (2008b). Resolution dependence of eddy fluxes. In M. W. Hecht, \& H. Hasumi (Eds.), Ocean modeling in an eddying regime (pp. 101-114). AGU volume 177 of Geophysical monograph.

Thoppil, P. G., Richman, J. G., \& Hogan, P. J. (2011). Energetics of a global ocean circulation model compared to observations. Geophys. Res. Lett., 38 , L15607. 
855 Urakawa, L. S., \& Hasumi, H. (2009). The energetics of global thermohaline 856 circulation and its wind-enhancement. J. Phys. Oceanogr., 39, 1715-1728. ${ }_{857}$ Urakawa, L. S., \& Hasumi, H. (2012). Eddy-resolving model estimate of the ${ }_{858}$ cabbeling effect on the water mass transformation in the Southern Ocean. $859 \quad$ J. Phys. Oceanogr., 42, 1288-1302.

860 Walin, G. (1982). On the relation between sea-surface heat flow and thermal 861 circulation in the ocean. Tellus, 34, 187-195. 